\title{
RD Optimized, Adaptive, Error-Resilient Transmission of MJPEG2000-Coded Video over Multiple Time-Varying Channels
}

\author{
Scott Bezan and Shahram Shirani \\ Department of Electrical and Computer Engineering, McMaster University, Hamilton, ON, Canada L8S 4K1
}

Received 25 February 2005; Revised 22 August 2005; Accepted 1 September 2005

To reliably transmit video over error-prone channels, the data should be both source and channel coded. When multiple channels are available for transmission, the problem extends to that of partitioning the data across these channels. The condition of transmission channels, however, varies with time. Therefore, the error protection added to the data at one instant of time may not be optimal at the next. In this paper, we propose a method for adaptively adding error correction code in a rate-distortion (RD) optimized manner using rate-compatible punctured convolutional codes to an MJPEG2000 constant rate-coded frame of video. We perform an analysis on the rate-distortion tradeoff of each of the coding units (tiles and packets) in each frame and adapt the error correction code assigned to the unit taking into account the bandwidth and error characteristics of the channels. This method is applied to both single and multiple time-varying channel environments. We compare our method with a basic protection method in which data is either not transmitted, transmitted with no protection, or transmitted with a fixed amount of protection. Simulation results show promising performance for our proposed method.

Copyright () 2006 Hindawi Publishing Corporation. All rights reserved.

\section{INTRODUCTION}

Video data is very large in its raw form and as such requires some level of source coding (compression) in order to be effectively transmitted. Unfortunately, many compression methods (usually of the lossy variety) introduce distortion in the reconstructed frame. In addition to the quantization distortion, source coding can leave the coded data vulnerable to bit errors that frequently occur in transmission over error-prone channels. If a constant source rate is assumed, any further distortion seen in the reconstructed frame will be due to channel-introduced errors. To this end, error protection (channel coding) is introduced to the source-coded data in order to enable correction of some erroneous bits at the receiver. Ideally, the channel coder could add as much channel code as necessary to correct all bit errors in transmission. In reality, transmission channels are bandlimited, so this solution is infeasible. Therefore, a rate-distortion (RD) tradeoff exists between the amount of error correction added, while considering a channel bandwidth constraint and the reconstructed distortion influenced by channel errors. Finding the optimum balance between rate and distortion is challenging, but more complication arises when channels are time-varying. In this case, when the condition of a channel changes with time, past optimized channel codes may no longer be relevant, therefore some manner of adaptation to the channel condition is required.

In discussing RD optimization of a bitstream transmitted over an error-prone channel, it is advantageous to consider blocks of the original data stream. These blocks, or coding units, divide a normally large amount of data into more manageable units. Also, in situations involving multiple channels, if channel resources are not sufficient for transmitting the entire bitstream, one can divide the bitstream using these coding units over multiple channels. Unfortunately, the dependence of one coding unit on another creates a challenge that must be addressed as errors occurring in presently decoded coding units will affect the decoding of future ones.

Over the past few years, many researchers have examined the problem of RD optimization of multimedia data. In [1], joint source-channel coding (JSCC) was performed on a wavelet-based source-coded bitstream using the bit sensitivities of the wavelet coefficients. An operational RD function was then constructed based on rate-compatible punctured convolutional (RCPC) channel codes. Unequal error protection was employed in [2] on a layered source coding scheme using Reed-Solomon codes to achieve JSCC. An iterative approach was used to find the appropriate source and channel code rates across a binary symmetric channel. JSCC was also 
employed in [3] using a progressive source coder and RCPC channel codes to find an exact solution for optimal channel code allocation.

In [4], JSCC was employed using a progressive wavelet source coder and the concatenation of RCPC and a cyclic redundancy-check code for channel error protection. The bitstream was transmitted over a binary symmetric channel and it was stated that the only effect that channel noise had on the system was degradation due to lower-rate source coding. Punctured turbo codes were used in [5] in conjunction with the JPEG2000 source coding standard to employ JSCC over a single binary symmetric channel. JSCC was performed to yield packets of fixed size resulting in a rate-allocation problem that grows exponentially with the number of packets to be transmitted.

New algorithms were proposed in [6] to find optimal solutions to uneven error protection problems involving scalable source-coded bitstreams. It was shown in [6] that the complexity of their algorithm decreases dramatically if information of the convexity of the source coder is known. JSCC was employed using a concatenation of Reed-Solomon, RCPC, and cyclic redundancy-check codes. The SPIHT encoder and RCPC codes were used by [7] for source and channel coding, respectively, and transmitted the protected bitstream over a time-varying channel. A method of subsampling the SPIHT source encoder was proposed by [8] to achieve multiple descriptions of the original data.

In summary, [1-8] performed JSCC on data to be transmitted over a single channel. In this paper, channel code was added in a forward error correction manner to preencoded source data transmitted over multiple time-varying channels. A multicast scenario was discussed in [9], however, our paper considers multiple-channel transmission to a single user.

Forward error correction was performed by [10] under various channel conditions, however, the amount of channel code added was fixed to the particular channel condition. In [11], forward error correction was added to various channel conditions using a dynamic programming approach, however, it was performed on mean packet loss rates, not a timevarying channel situation.

In this paper, JPEG2000 is applied using frames of video data, appended with channel code and transmitted over multiple error-prone, time-varying channels. The channel coder chosen is a rate-compatible punctured convolutional (RCPC) coder which allows the generation of multiple code rates from a single encoder. Analysis is performed in order to determine the RD optimal amount of channel code to add to the source-coded frame based on the condition of the time-varying channel(s). As JPEG2000 compression is performed using frames of a video sequence, this is analogous to Motion JPEG2000 (MJPEG2000). In terms of compression efficiency, other video compression standards outperform MJPEG2000 due to the use of motion-compensated prediction between frames. However, MJPEG2000 encodes each frame independent of others and therefore offers superior performance when considering video editing and error robustness [12]. In addition, MJPEG2000 makes use of the wavelet transform which adds the advantage of a lower

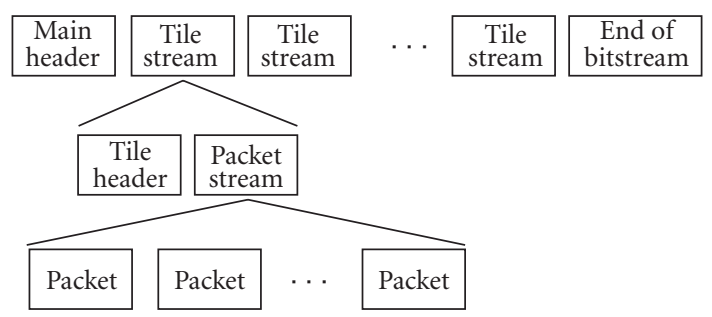

FIgURe 1: JPEG2000 bitstream [15].

computational complexity than the calculation and manipulation of motion vectors used in MPEG video compression [13].

Lastly, an argument could be made that a comparison between the approach proposed in this paper and multiple description coding with path diversity would be needed. Unfortunately, this was difficult due to the lack of research performed on multiple description coding over time-varying channels.

The paper is organized as follows. Section 2 discusses the JPEG2000 source and RCPC channel coder and channel model used herein. Section 3 discusses the proposed method of the paper by introducing the rate-distortion optimization techniques used. Next, experimental results for transmission across both single and multiple time-varying channels are outlined in Section 4. Finally, Section 5 concludes the paper.

\section{BACKGROUND}

\subsection{Source coding}

The JPEG2000 image compression standard was created to overcome the drawbacks of the existing widely used JPEG standard [10]. MJPEG2000 is the extension to video coding of the JPEG2000 standard where more attention is payed to error robustness and for the purpose of use in highquality video systems [14]. Conversely, in MPEG video coding where compression efficiency is paramount, interframe coding (as well as intra-frame coding) is realized using motion-compensated prediction resulting in the propagation of errors throughout the decoded video sequence due to the interframe dependencies. In MJPEG2000, each frame is intra-coded without any dependency of surrounding frames. If errors occur in transmission, they will be confined to the frame in which they occurred [13].

Frames in MJPEG2000 are first divided into user-defined, nonoverlapping rectangular subframes called tiles which are coded independently using a combination of wavelets, quantization, and arithmetic encoding. The final JPEG2000 bitstream results in a sequence of headers detailing the encoding process, followed by the compressed data (see Figure 1). Following the main header are the independently coded tile streams. Each tile stream is comprised of a tile header and a stream of packets. The stream of packets is a packetized version of the compressed data [15]. Finally, the bitstream is concluded with a header indicating the end of the coded data. 


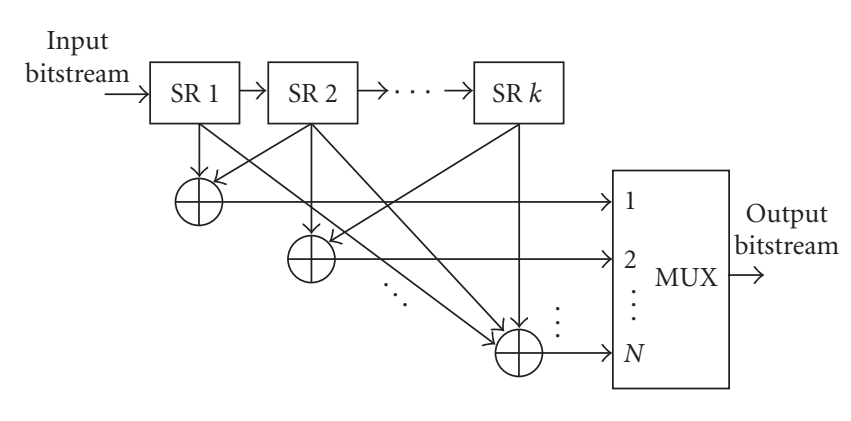

Figure 2: Basic convolutional encoder.

From the JPEG2000 encoder, two types of coding units are used here: tiles and packets. When tiles are the coding unit in question, the original frame will be encoded into a number of tiles and the packet stream in each tile is limited to one packet. Conversely, when packets are used, the entire frame is encoded as one large tile and multiple packets.

\subsection{Channel coding}

Channel coding is imperative when source-coded data is to be transmitted across error-prone channels. Convolutional codes are a type of channel code that interleaves redundant data into the bitstream so that erroneous bits can be corrected at the receiver. A convolutional encoder combines the present input bit with $N$ combinations of up to $k-1$ past input bits (Figure 2). The $N$ output bits are multiplexed to yield a continuous bitstream. A binary generator matrix $\mathbf{G}$ of size $k \times N$ details the connectivity between the input bits and the $N$ output bits.

To achieve multiple rates from a single encoder, an $N \times$ $P$ puncture matrix is inserted between the $N$ output substreams and the multiplexer (not represented in Figure 2), where $P$ is denoted as the "puncturing period." Blocks of $P$ bits from the $N$ output substreams are clocked into the puncture matrix. Wherever a "0" appears in the matrix, the corresponding bit in that position of the substream is discarded. Therefore, from an original code rate of $1 / N$, multiple rates can be generated. In (1), $r_{c}$ is the channel coding rate and $1 \leq z \leq(N-1) P$, where $z$ is a parameter that is indicative of the amount of puncturing [16]:

$$
r_{c}=\frac{P}{P+z} \text {. }
$$

Introducing a rate-compatibility restriction on the puncture matrices ensures a mapping between different code rates. For a set of puncture matrices $p(z)$, the rate-compatibility restriction states that codes of a high code rate are embedded in those of a low code rate. This is detailed in [16] as follows:

$$
\text { if } p_{i j}\left(z_{o}\right)=1, \quad \text { then } p_{i j}(z)=1 \quad \forall z \geq z_{o}, z_{o} \geq 1
$$

or, equivalently,

$$
\text { if } p_{i j}\left(z_{o}\right)=0, \quad \text { then } p_{i j}(z)=0 \quad \forall z \leq z_{0}, z_{0} \leq(N-1) P-1 \text {, }
$$

where $i$ and $j$ are indices for the rows and columns of $p(z)$, respectively.

\subsection{Channel model}

Practical transmission channels are rarely static in terms of their characteristics. Since this paper examines the situation where the bit error rate of transmission channels varies with time, channels were simulated using additive white Gaussian noise (AWGN) to introduce random bit errors and an autoregressive model was used to simulate time-varying nature of the channel's characteristics. The autoregressive model used was as follows:

$$
\operatorname{BER}(n)=a \operatorname{BER}(n-1)+w(n),
$$

where BER denotes the bit error rate, $n$ is the time index, $a$ is a correlation factor, and $w(n)$ is a factor of white Gaussian noise. Therefore, the BER at the current time index is based on the most current past BER, weighted by the factor $a$ and white Gaussian noise $w(n)$. A similar model was used in $[17,18]$ to characterize time-varying channels. As the BER changes by (4), the amount of error correction code should adapt accordingly.

\section{PROPOSED ANALYSIS METHOD}

Figure 3 shows the block diagram of the system used in this paper. The raw frame data (only the Y-component of the YUV sequence was utilized) is fed into the source coding block. Here, the raw frame is encoded at a constant rate into a number of coding units using MJPEG2000. After the completion of the source encoding, the coding units are then channel coded using an RCPC coder. To find the optimum amount of channel code to add at a particular instant of time, the RD optimization block polls the channels for their particular condition. With this information, the RD optimization block performs an analysis on the coding units of the frame. This analysis yields the appropriate amount of channel code to add to the coding units in order to minimize the reconstructed distortion while maintaining a rate budget dictated by the channel(s) and a transmission delay. On the receiver side, the received data is first channel decoded using the Viterbi maximum likelihood algorithm, then source decoded using the MJPEG2000 decoder. We show next how to adaptively optimize the amount of error correction code added to a video source-coded bitstream based on the conditions of time-varying channels. The details of the proposed analysis method are as follows.

As mentioned previously, an RCPC coder is used as the channel coder. Assume $M$ puncture matrices $\mathbf{p}$ each yielding a particular amount of protection. Each coding unit $i$ can then be encoded into $M$ possible rates $\mathbf{r}_{i}$. The coding units encoded at a particular rate will have an associated distortion $\mathbf{d}_{i}$.

$$
\begin{aligned}
\mathbf{p} & =\{p(1), \ldots, p(M)\} \\
\mathbf{r}_{i} & =\left\{r_{i, p(1)}, \ldots, r_{i, p(M)}\right\} \\
\mathbf{d}_{i} & =\left\{d_{i, p(1)}, \ldots, d_{i, p(M)}\right\}
\end{aligned}
$$




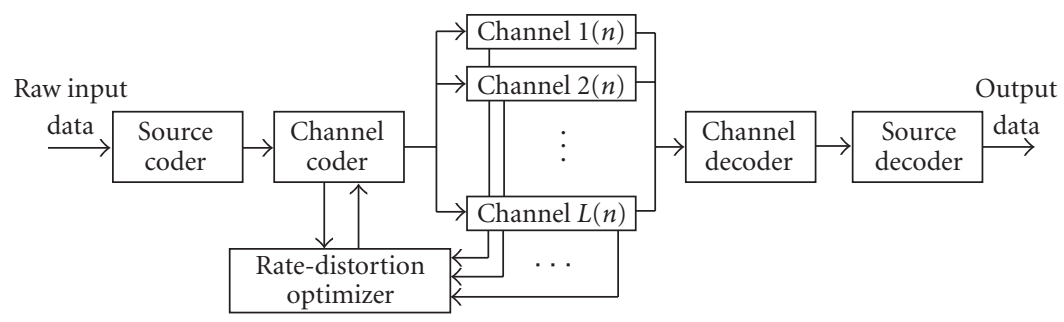

Figure 3: System diagram.

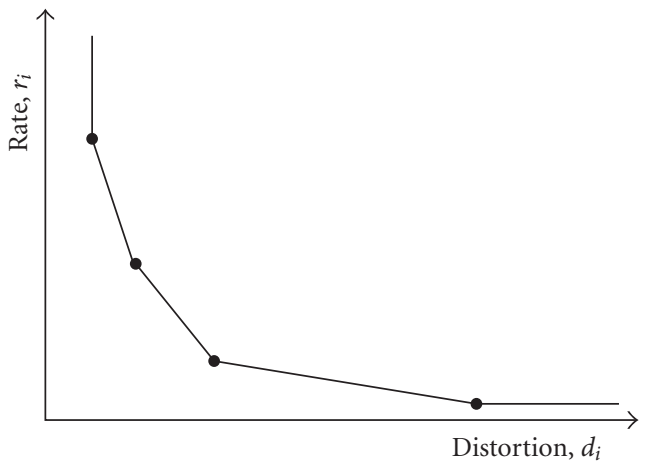

FIGURE 4: Rate-distortion map of operating points, where $i$ represents the $i$ th coding unit.

The pairs $\left(r_{i, p(m)}, d_{i, p(m)}\right), m=1, \ldots, M$, create operating points on an RD map for the ith coding unit (Figure 4). Assuming that the source coder encodes the data into $T$ coding units, an optimal bit allocation can be performed to yield a minimum reconstructed distortion while maintaining a bandwidth constraint. An optimal result can be found using dynamic programming as in $[5,6]$ as well as the Lagrangian method as in $[2,19]$. The Lagrangian method was preferred for its ease of implementation and computational advantages [20].

\subsection{Independent coding units}

Assuming $T$ independent coding units (that can be coded independently), the total distortion is calculated as

$$
D=\sum_{i=1}^{T} d_{i, p\left(m_{i}\right)}
$$

where $p\left(m_{i}\right)$ is a selected puncture matrix from the set $\{p(1), \ldots, p(M)\}$ for coding unit $i$. We wish to minimize the total distortion subject to a channel defined rate constraint, that is,

$$
\min D \quad \text { subject to } R=\sum_{i=1}^{T} \leq R_{c}
$$

In the above equation, $R_{c}=B \cdot \tau_{m}$ is the channel rate constraint, $B$ is the bandwidth in (bps), and $\tau_{m}$ is the maximum acceptable delay in transmission of the frame. The transmission delay $\tau$ is defined as the amount of time it takes to transmit $T$ coding units over a channel with a certain bandwidth of $B$ bps and it is given by

$$
\tau=\frac{\sum_{i=1}^{T} r_{i, p\left(m_{i}\right)}}{B},
$$

where

$$
r_{i, p\left(m_{i}\right)}=\frac{r_{s, i}}{r_{c, i}}
$$

and $r_{s, i}$ is the rate in bits for the $i$ th coding unit available from the source coder. $r_{c, i}$ is the channel code rate based on $p\left(m_{i}\right)$, where the subscript $i$ is added to $r_{c}$ to indicate the channel coding rate for coding unit $i$.

Using Lagrangian optimization, we can define a Lagrangian cost function $J_{i}$ for each coding unit given by

$$
J_{i}=d_{i, p\left(m_{i}\right)}+\lambda r_{i, p\left(m_{i}\right)} .
$$

We can now change the optimization problem of (7) to

$$
\min \sum_{i=1}^{T} J_{i}=\sum_{i=1}^{T} \min J_{i},
$$

where we have used the fact that the cost functions for each coding unit can be optimized independently [20]. Graphically, the above method translates to finding a line of slope $\lambda$ that is tangent to the convex hull of the RD point $\left(r_{i, p(m)}, d_{i, p(m)}\right)$ for each coding unit. Analysis is performed by calculating the sum of the rates $r_{i, p(m)}$ from the operating points for all coding units $i=1, \ldots, T$. If the sum is greater than the rate budget, then $\lambda$ must be altered to yield a smaller rate summation. Therefore, the reduction in distortion from using an extra bit of channel code for one coding unit is equal to the increase of distortion seen at another [20]. This analysis is performed recursively until the optimal balance is found.

\subsection{Dependent coding units}

If coding units are dependent on one another, errors from one decoded block of data will transfer to future decoded coding units. The total distortion is then given by

$$
D=\sum_{i=1}^{T} d_{i, p\left(m_{i}\right) \mid p\left(m_{i-1}\right), \ldots, p\left(m_{1}\right)},
$$




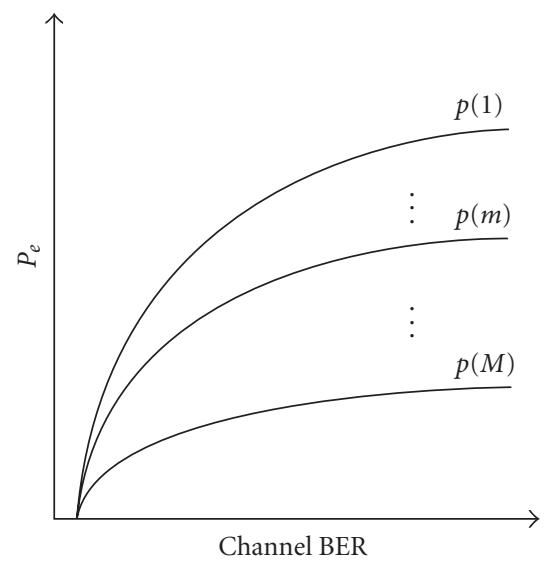

Figure 5: Channel characteristic plot.

where $d_{i, p\left(m_{i}\right) \mid p\left(m_{i-1}\right), \ldots, p\left(m_{1}\right)}$ is the distortion of the $i$ th coding unit based on the protection used on coding units $1,2,3, \ldots, i-1$. The rate constraint of (7) remains the same, however, the Lagrange cost functions must reflect the conditional distortion:

$$
\begin{gathered}
J_{1}(\lambda)=d_{1, p\left(m_{1}\right)}+\lambda r_{1, p\left(m_{1}\right)}, \\
J_{2}(\lambda)=d_{2, p\left(m_{2}\right) \mid p\left(m_{1}\right)}+\lambda r_{2, p\left(m_{2}\right)} \\
\vdots \\
J_{T}(\lambda)=d_{T, p\left(m_{T}\right) \mid p\left(m_{T-1}\right), \ldots, p\left(m_{1}\right)}+\lambda r_{T, p\left(m_{T}\right)} .
\end{gathered}
$$

From (12), it is clear that the distortion at any coding unit $i$ depends on the amount of protection used for previous coding units $i=1, \ldots, T$. Obviously, finding all possible combinations of distortions for all coding units is too complex. To alleviate this problem, we used the universal rate-distortion characteristic (URDC) of $[19,21]$.

Given a multiple rate channel coder and information of the channel, we can calculate how the channel code will perform in a particular channel environment. Assume a set of puncture matrices $p=\{p(1), \ldots, p(M)\}$ which create a set of code rates $r_{c}=\left\{r_{c, p(1)}, \ldots, r_{c, P(M)}\right\}$. If we know the condition of a channel in terms of its BER, we can perturb a string of data protected with each aforementioned puncture matrix. We then channel decode the perturbed data and calculate the effective probability of bit error $\left(P_{e}\right)$ for each amount of protection. This gives us an idea of how the channel codes will perform in this particular noisy environment. If the channel condition is known to be in a specific range, a set of $P_{e}$ can be calculated for each BER and the results can be stored in a lookup table called a channel characteristic plot (see Figure 5). These calculations can be performed offline to reduce the amount of computational complexity at run time.

Since we are considering a constant source rate encoder, the distortion for any coding unit $i$ is a function of $P_{e}$ after channel decoding. A family of curves describing the distortion for a particular coding unit $d_{i}$ versus the inverse $P_{e}$ 's is called the URDC curve [19]. It is extremely difficult to analytically obtain the URDCs due to application of variable length codes. Therefore, URDCs are obtained using simulations. These curves are generated by perturbing a coding unit $i$ with bit error $P_{e}$ (found using the current state of the channel and the channel characteristic plot) while coding units $1,2, \ldots, i-1, i+1, \ldots, T$ are not corrupted. After the coding units are subjected to the appropriate bit errors, the resultant bitstreams are decoded and their reconstructed distortions are measured (this method is detailed in Figure 6). This process is ensemble averaged over a number of average iterations (e.g., 25 times). More details on URDC can be found in $[19,21]$ and a pictorial version is shown in Figure 6.

Considering the protections given to previous coding units and the individual contribution to distortion from coding unit $i$ found using the URDC and channel characteristic plot, we now have a set of distortions and rates necessary to create the dependent RD map for coding unit $i$. Once this is done for each coding unit, the Lagrange cost functions are built and the optimization is performed as in Section 3.1.

\subsection{Multiple time-varying channels}

Assume now that $L$ channels are available for transmission of the string of $T$ coding units. It is further postulated that no single channel can handle all $T$ coding units. The problem then becomes how to allocate the coding units across the channels of varying condition such that the reconstructed frames have minimum distortion and all rate limitations of the channels are respected. The order of the channels in terms of quality is assumed to be unimportant. This is a fair assumption since the decoder is uninterested in the avenue through which the data was sent.

The transmission delay in the multiple channel case is as follows. Let $r_{i}$ denote the rate of the $i$ th coding unit after error correction code. Then, in order to transmit $T$ coding units, the set $\left\{r_{1}, r_{2}, \ldots, r_{T}\right\}$ is partitioned into $L$ sets $C_{1}, C_{2}, \ldots, C_{L}$, where $C_{j}=\left\{r_{i} \mid\right.$ coding unit $i$ is transmitted over channel $\left.j\right\}$. Then the transmission delay will be equal to

$$
\tau=\max _{j}\left(\frac{\sum_{l \in C_{j}} r_{l}}{B_{j}}\right)
$$

where $B_{j}$ is the bandwidth of the $j$ th channel (in bps).

The method for RD optimization across multiplechannels is much the same as optimizing one channel. In this case, however, an initial $\lambda$ is selected so as to return a large rate summation. The coding units are now to be partitioned among the channels. For this, a greedy algorithm is employed. Coding units are allocated to the first available channel until the rate budget can no longer accommodate another coding unit. This channel is then considered closed and coding units are scheduled to be transmitted through the next available channel. This process continues until either the last coding unit is scheduled, or the rate constraints of all channels are exceeded and coding units remain. In the case of the latter, $\lambda$ must be adjusted to allow a lower rate summation. 


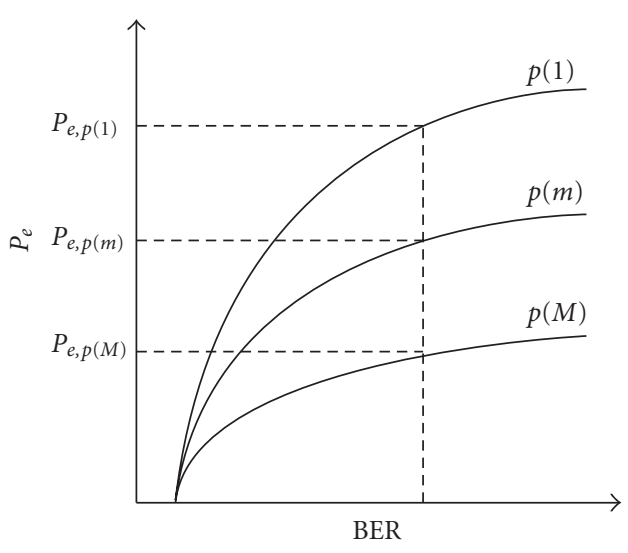

(a)

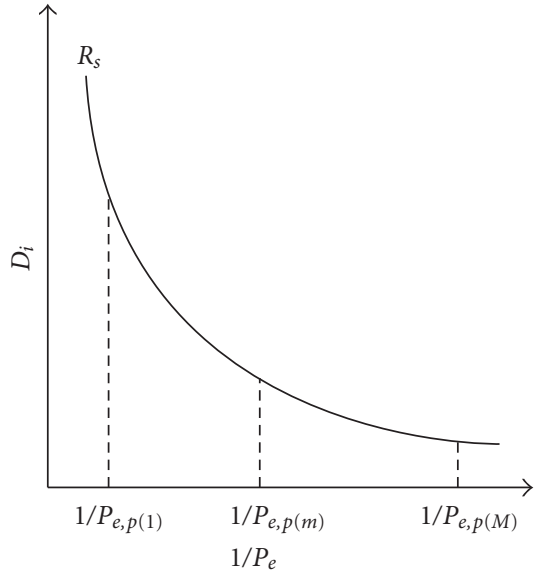

(b)

FIGURE 6: Method for finding distortion of dependent coding units. Figure (a) represents the channel characteristic plot and (b) represents the universal rate-distortion characteristic.

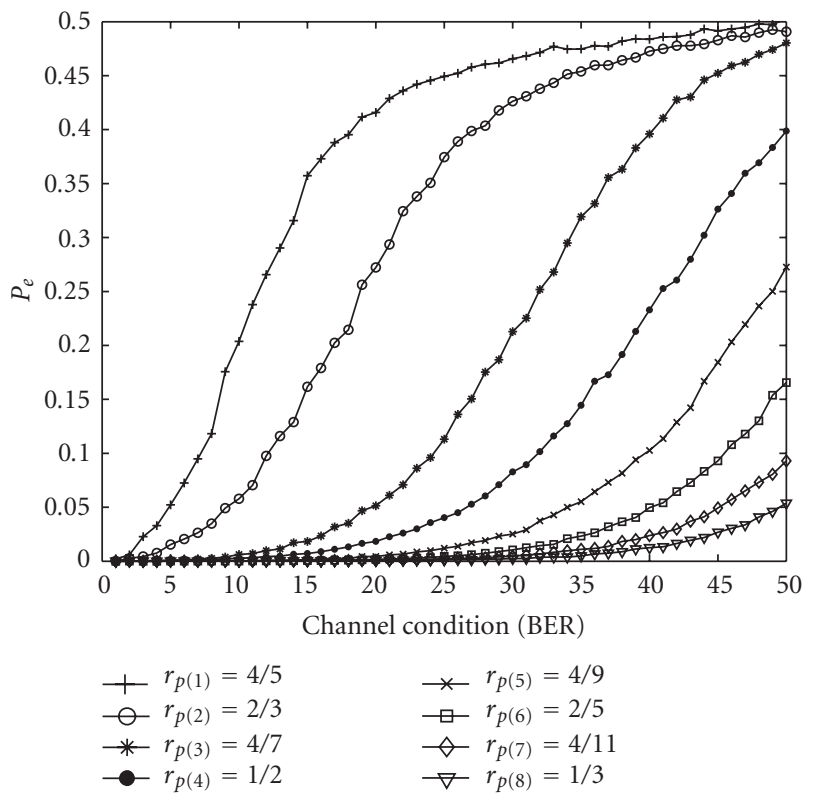

FIGURE 7: Channel characteristic plot for AR(1) channel with $a=$ 0.5 .

\section{EXPERIMENTAL RESULTS}

Frames from the sequences Mobile and Paris (each of size CIF or $352 \times 288$ pixels) were used to test the proposed method. Frames 100, 101, and 102 from each sequence were encoded using David Taubman's JPEG2000 encoder version 2.2 at 0.5 bit per pixel (bpp). Using tiles as coding units, the frames were encoded into 30 tiles. Conversely, when packets were used as coding units, 17 packets were generated.

\subsection{Protection scheme}

For the purpose of analysis, we compare our adaptive protection (AP) approach with a basic protection (BP) scheme. In the AP scheme, the amount of error correction code added to a coding unit is reoptimized at each new time interval based on the condition of the channel(s). The BP scheme, however, creates a set of RD curves with only three operating points.

(1) The coding unit is dropped.

(2) The coding unit is transmitted unprotected.

(3) The coding unit is protected with a predefined amount of error correction code.

This family of RD curves is then searched exhaustively to find the optimal solution for basic protection. This BP scheme was used as we were unable to find previous works with which to compare the time-varying channel model employed for frames.

The RCPC encoder had an unpunctured rate of $1 / 3$, a constraint length of $k=5$, and a generator matrix $\mathbf{G}=\left[\begin{array}{lll}23 & 35 & 57\end{array}\right]^{T}$ represented in octal. Using puncture matrices found in [16], channel coding rates of $r_{c}=\{4 / 5,2 / 3,4 / 7,1 / 2,4 / 9,2 / 5,4 / 11,1 / 3\}$ were available. The constant protection of the BP scheme had a rate equal to $r_{c, \mathrm{BP}}=4 / 9$.

As indicated in Section 2.3, the time-varying nature of the channels was modeled using a first-order AR process. The value of the parameter in (4) was $a=0.5$. The nature of the channel by way of the channel characteristic plot is seen in Figure 7.

\subsection{Results for tiles}

The bitstreams were divided into 30 tiles. Based on the size of the frames $(352 \times 288$ pixels $)$, there are 4 sizes of tiles. The tile in the bottom-right corner of the frame will be $46 \times 52$ pixels. Secondly, the tiles along the right edge of the frame (excluding the aforementioned corner) are $60 \times 52$ pixels. The tiles along the bottom edge of the frame (excluding the lower-right corner) are of size $46 \times 60$ pixels. Finally, the remaining tiles are $60 \times 60$ pixels. Tables 1,2 , and 3 show the 
TABLE 1: PSNR (dB) values for tiles over a single time-varying channel of indicated bandwidth.

\begin{tabular}{|c|c|c|c|c|c|c|}
\hline \multirow{3}{*}{ Sequence } & \multirow{3}{*}{$\tau(\mathrm{s})$} & \multicolumn{4}{|c|}{ B (bps) } & \multirow{3}{*}{$\begin{array}{l}\text { Frame } \\
\text { number }\end{array}$} \\
\hline & & \multicolumn{2}{|c|}{25000} & \multicolumn{2}{|c|}{30000} & \\
\hline & & $\mathrm{AP}$ & $\mathrm{BP}$ & $\mathrm{AP}$ & $\mathrm{BP}$ & \\
\hline \multirow{6}{*}{ Mobile } & \multirow{3}{*}{10} & 21.880 & 20.856 & 21.889 & 20.921 & 100 \\
\hline & & 21.890 & 21.209 & 21.892 & 21.240 & 101 \\
\hline & & 21.904 & 21.212 & 21.899 & 20.849 & 102 \\
\hline & \multirow{3}{*}{6} & 21.839 & 20.771 & 21.887 & 20.774 & 100 \\
\hline & & 21.850 & 20.952 & 21.874 & 20.508 & 101 \\
\hline & & 21.897 & 21.099 & 21.889 & 20.669 & 102 \\
\hline \multirow{6}{*}{ Paris } & \multirow{3}{*}{10} & 25.790 & 23.970 & 25.789 & 23.793 & 100 \\
\hline & & 25.811 & 24.563 & 25.819 & 24.409 & 101 \\
\hline & & 25.804 & 24.470 & 25.801 & 24.151 & 102 \\
\hline & \multirow{3}{*}{6} & 25.658 & 23.499 & 25.788 & 23.212 & 100 \\
\hline & & 25.766 & 23.981 & 25.770 & 23.831 & 101 \\
\hline & & 25.744 & 24.318 & 25.676 & 23.706 & 102 \\
\hline
\end{tabular}

TABLE 2: PSNR ( $\mathrm{dB})$ values for tiles over two time-varying channels of indicated bandwidth.

\begin{tabular}{|c|c|c|c|c|c|c|}
\hline \multirow{3}{*}{ Sequence } & \multirow{3}{*}{$\tau(\mathrm{s})$} & \multicolumn{4}{|c|}{ B (bps) } & \multirow{3}{*}{$\begin{array}{l}\text { Frame } \\
\text { number }\end{array}$} \\
\hline & & \multirow{2}{*}{\multicolumn{2}{|c|}{$\begin{array}{cc}10000, & 20000 \\
\mathrm{AP} & \mathrm{BP} \\
\end{array}$}} & \multicolumn{2}{|c|}{20000,18000} & \\
\hline & & & & $\mathrm{AP}$ & $\mathrm{BP}$ & \\
\hline \multirow{6}{*}{ Mobile } & \multirow{3}{*}{10} & 21.870 & 20.321 & 21.887 & 20.517 & 100 \\
\hline & & 21.880 & 20.190 & 21.886 & 20.989 & 101 \\
\hline & & 21.890 & 19.883 & 21.904 & 21.115 & 102 \\
\hline & \multirow{3}{*}{6} & 21.855 & 20.028 & 21.832 & 20.084 & 100 \\
\hline & & 21.283 & 19.981 & 21.836 & 19.971 & 101 \\
\hline & & 21.559 & 19.501 & 21.856 & 20.880 & 102 \\
\hline \multirow{6}{*}{ Paris } & \multirow{3}{*}{10} & 25.735 & 22.555 & 25.778 & 23.356 & 100 \\
\hline & & 25.783 & 23.104 & 25.817 & 23.338 & 101 \\
\hline & & 25.781 & 23.032 & 25.764 & 23.416 & 102 \\
\hline & \multirow{3}{*}{6} & 24.237 & 21.390 & 25.731 & 22.649 & 100 \\
\hline & & 24.759 & 22.660 & 25.749 & 21.260 & 101 \\
\hline & & 24.708 & 21.615 & 25.701 & 22.582 & 102 \\
\hline
\end{tabular}

PSNR values for transmission of tiles over 1, 2, and 3 timevarying channels, respectively. In all cases, the PSNR of the AP scheme outperforms that of the BP scheme. In addition, when the amount of transmission delay is reduced, the PSNR values are less than or equal to those of longer delay. This is due to the fact that more coding units were transmitted in a set amount of time thereby spreading out the available resources across more data.

Figures 8, 9, and 10 show frames 100, 101, and 102 (vertical columns) visually detailing the performance of the AP scheme over the BP scheme. For example, frame 100 in Figures $8(\mathrm{a})$ and $8(\mathrm{~d})$, the ball in the lower middle of the frame is distorted in the BP frame and not as much in the AP frame. It should be noted that since the simulations were run over 25 iterations (on average), the frames chosen are those for which the PSNR of the frame best matches (in a mean-square sense) the mean PSNR over the average number of iterations.
TABLE 3: PSNR (dB) values for tiles over three time-varying channels of indicated bandwidth.

\begin{tabular}{|c|c|c|c|c|c|c|}
\hline \multirow{3}{*}{ Sequence } & \multirow{3}{*}{$\tau(\mathrm{s})$} & \multicolumn{4}{|c|}{ B (bps) } & \multirow{3}{*}{$\begin{array}{l}\text { Frame } \\
\text { numbe }\end{array}$} \\
\hline & & \multicolumn{2}{|c|}{$15000,9000,1000$} & \multicolumn{2}{|c|}{$30000,25000,15000$} & \\
\hline & & $\mathrm{AP}$ & BP & $\mathrm{AP}$ & BP & \\
\hline \multirow{6}{*}{ Mobile } & \multirow{3}{*}{10} & 21.897 & 20.412 & 21.896 & 21.234 & 100 \\
\hline & & 21.893 & 20.590 & 21.893 & 21.324 & 101 \\
\hline & & 21.891 & 20.805 & 21.905 & 21.193 & 102 \\
\hline & \multirow{3}{*}{6} & 21.748 & 20.260 & 21.878 & 20.059 & 100 \\
\hline & & 21.683 & 20.470 & 21.875 & 21.011 & 101 \\
\hline & & 21.308 & 20.322 & 21.901 & 20.962 & 102 \\
\hline \multirow{6}{*}{ Paris } & \multirow{3}{*}{10} & 25.800 & 23.804 & 25.800 & 24.128 & 100 \\
\hline & & 25.819 & 23.690 & 25.819 & 24.643 & 101 \\
\hline & & 25.738 & 22.239 & 25.804 & 24.496 & 102 \\
\hline & \multirow{3}{*}{6} & 24.952 & 22.869 & 25.800 & 24.035 & 100 \\
\hline & & 25.480 & 23.572 & 25.783 & 23.175 & 101 \\
\hline & & 25.238 & 22.211 & 25.801 & 24.032 & 102 \\
\hline
\end{tabular}

TABLE 4: PSNR ( $\mathrm{dB}$ ) values for packets over a single time-varying channel of indicated bandwidth.

\begin{tabular}{|c|c|c|c|c|c|c|}
\hline \multirow{3}{*}{ Sequence } & \multirow{3}{*}{$\tau(\mathrm{s})$} & \multicolumn{4}{|c|}{ B (bps) } & \multirow{3}{*}{$\begin{array}{l}\text { Frame } \\
\text { number }\end{array}$} \\
\hline & & \multicolumn{2}{|c|}{45000} & \multicolumn{2}{|c|}{50000} & \\
\hline & & AP & $\mathrm{BP}$ & $\mathrm{AP}$ & BP & \\
\hline \multirow{6}{*}{ Mobile } & \multirow{3}{*}{5.67} & 16.160 & 12.830 & 18.840 & 14.678 & 100 \\
\hline & & 17.383 & 13.043 & 18.130 & 13.663 & 101 \\
\hline & & 15.831 & 12.579 & 17.654 & 14.461 & 102 \\
\hline & \multirow{3}{*}{3.4} & 11.141 & 10.679 & 12.706 & 11.929 & 100 \\
\hline & & 11.443 & 10.475 & 12.846 & 11.963 & 101 \\
\hline & & 11.693 & 10.586 & 11.984 & 11.604 & 102 \\
\hline \multirow{6}{*}{ Paris } & \multirow{3}{*}{5.67} & 21.887 & 17.270 & 24.141 & 23.511 & 100 \\
\hline & & 22.325 & 17.111 & 22.697 & 22.289 & 101 \\
\hline & & 20.309 & 17.409 & 24.518 & 24.486 & 102 \\
\hline & \multirow{3}{*}{3.4} & 14.180 & 13.690 & 17.732 & 13.704 & 100 \\
\hline & & 14.330 & 13.970 & 17.063 & 15.330 & 101 \\
\hline & & 13.998 & 13.505 & 15.769 & 13.851 & 102 \\
\hline
\end{tabular}

\subsection{Results for packets}

Much like the results for tiles, Tables 4, 5, and 6 show the PSNR values for transmitting packets over one, two, and three time-varying channels, respectively. Again, in all cases the AP scheme outperforms the BP scheme and as transmission delay changes from 5.67 seconds down to 3.4 seconds, the PSNR values decrease accordingly. Figures 11,12 and 13 show frames 100, 101 and 102 from video sequences Paris and Mobile illustrating the performance of the AP scheme over the BP scheme for the case of packets.

\subsection{Time adaptivity}

Figures 14,15 , and 16 show time adaptivity for data transmitted across one, two and three channels, respectively. In these figures graphs (a) show the selected puncture matrices, 


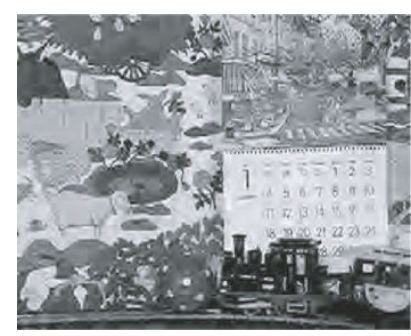

Frame 100

(a)

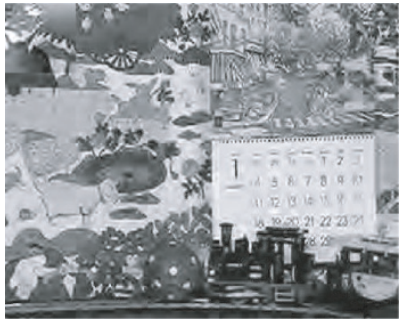

Frame 100

(d)

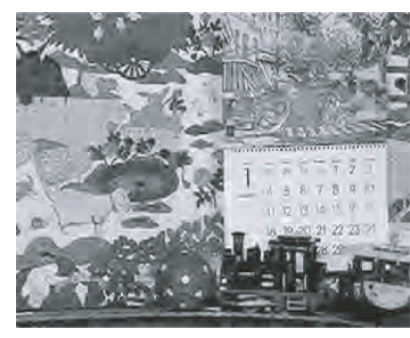

Frame 101

(b)

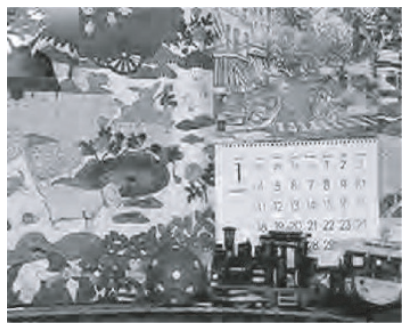

Frame 101

(e)

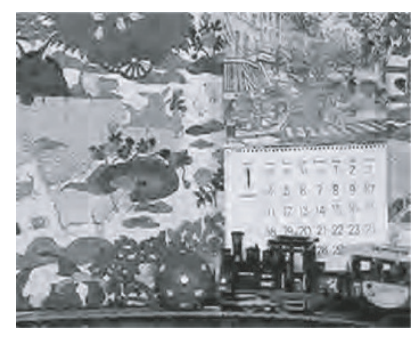

Frame 102

(c)

Figure 8: Decoded frames of Mobile sequence using (a), (b), (c) adaptive protection and (d), (e), (f) basic protection, all transmitted over a single channel with a bandwidth of $25000 \mathrm{bps}(\tau \sim 10 \mathrm{~s})$.

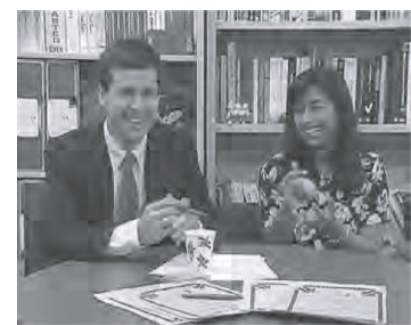

Frame 100

(a)

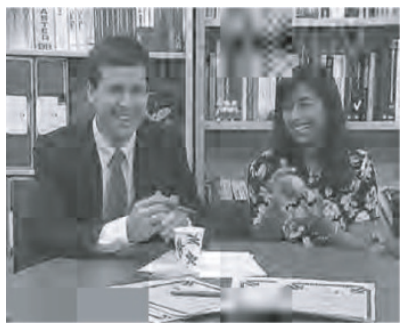

Frame 100

(d)

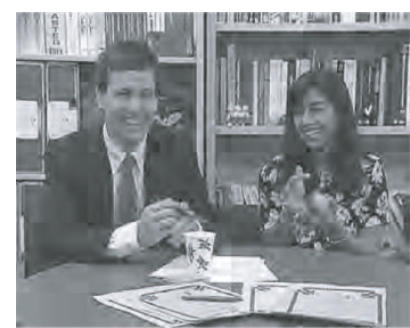

Frame 101

(b)

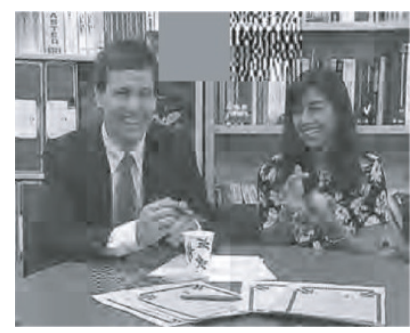

Frame 101

(e)

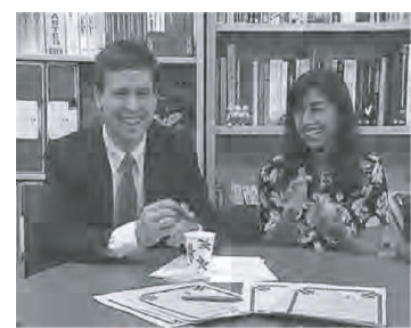

Frame 102

(c)

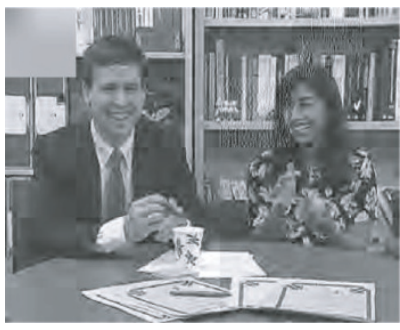

Frame 102

(f)

Figure 9: Decoded frames of Paris sequence using (a), (b), (c) adaptive protection and (d), (e), (f) basic protection, all transmitted over a single channel with bandwidths of 20000 and 18000 bps $(\tau \sim 6 \mathrm{~s})$. 


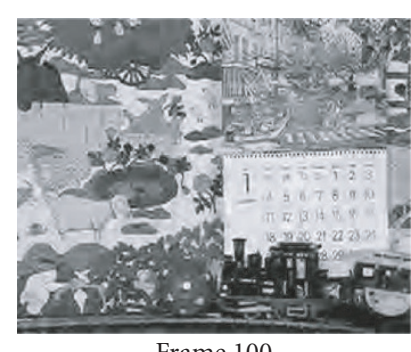

(a)

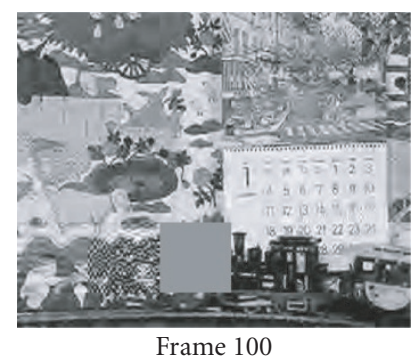

(d)

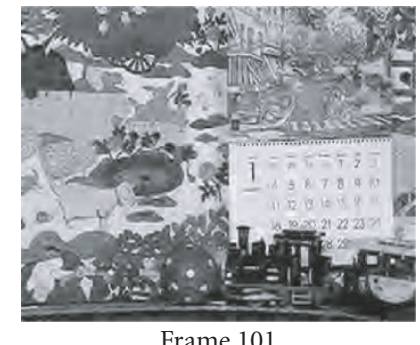

rame 10

(b)

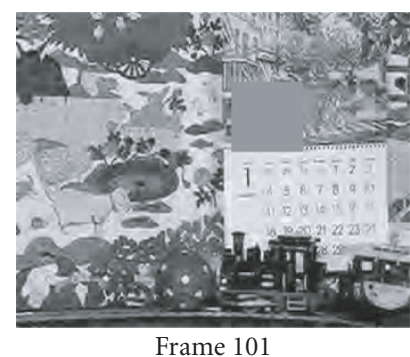

(e)

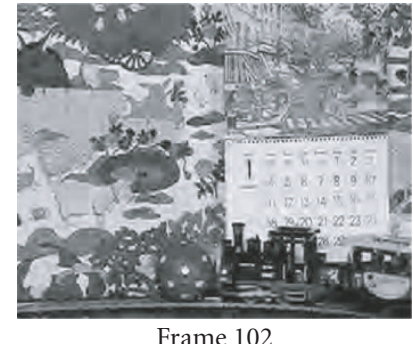

(c)

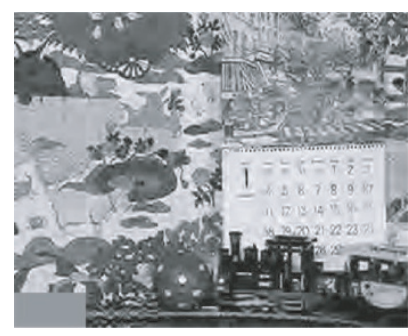

Frame 102

(f)

Figure 10: Decoded frames of Mobile sequence using (a), (b), (c) adaptive protection and (d), (e), (f) basic protection, all transmitted over a single channel with bandwidths of 30000,25000 , and 15000 bps $(\tau \sim 10$ s).

TABLE 5: PSNR (dB) values for packets over two time-varying channels of indicated bandwidth.

\begin{tabular}{|c|c|c|c|c|c|c|}
\hline \multirow{3}{*}{ Sequence } & \multirow{3}{*}{$\tau(\mathrm{s})$} & \multicolumn{4}{|c|}{ B (bps) } & \multirow{3}{*}{$\begin{array}{l}\text { Frame } \\
\text { number }\end{array}$} \\
\hline & & \multicolumn{2}{|c|}{42000,45000} & \multicolumn{2}{|c|}{57000,60000} & \\
\hline & & $\mathrm{AP}$ & BP & $\mathrm{AP}$ & BP & \\
\hline \multirow{6}{*}{ Mobile } & \multirow{3}{*}{5.67} & 22.237 & 20.498 & 22.824 & 20.560 & 100 \\
\hline & & 21.491 & 18.951 & 22.864 & 19.724 & 101 \\
\hline & & 22.291 & 19.234 & 23.007 & 20.389 & 102 \\
\hline & \multirow{3}{*}{3.4} & 21.105 & 19.120 & 22.537 & 18.248 & 100 \\
\hline & & 20.587 & 18.197 & 22.592 & 19.610 & 101 \\
\hline & & 20.265 & 18.566 & 22.999 & 19.407 & 102 \\
\hline \multirow{6}{*}{ Paris } & \multirow{3}{*}{5.67} & 27.288 & 22.445 & 27.511 & 23.448 & 100 \\
\hline & & 27.559 & 25.070 & 27.215 & 23.343 & 101 \\
\hline & & 27.437 & 21.998 & 27.506 & 21.632 & 102 \\
\hline & \multirow{3}{*}{3.4} & 26.517 & 20.909 & 27.183 & 21.358 & 100 \\
\hline & & 26.750 & 20.050 & 27.007 & 22.011 & 101 \\
\hline & & 26.341 & 20.908 & 27.371 & 20.432 & 102 \\
\hline
\end{tabular}

whereas graphs (b) show the BER of the channels. Figure 14 is an example of an average iteration for frame 100 of the Mobile sequence encoded into tiles and transmitted across a single channel with a bandwidth of 25000 bps. Figure 15 shows the timing situation for frame 100 of the Mobile sequence transmitted across two channels with bandwidths of 42000 and 45000 bps. Finally, Figure 16 shows frame 100 of the Mobile sequence transmitted across channels of 35000 , 30000 , and 32000 bps. It can be seen from these figures that the levels of protection used change in the same manner as
TABLE 6: PSNR ( $\mathrm{dB}$ ) values for packets over three time-varying channels of indicated bandwidth.

\begin{tabular}{|c|c|c|c|c|c|c|}
\hline \multirow{3}{*}{ Sequence } & \multirow{3}{*}{$\tau(\mathrm{s})$} & \multicolumn{4}{|c|}{ B (bps) } & \multirow{3}{*}{$\begin{array}{l}\text { Frame } \\
\text { number }\end{array}$} \\
\hline & & \multicolumn{2}{|c|}{$35000,30000,32000$} & \multicolumn{2}{|c|}{$50000,40000,35000$} & \\
\hline & & AP & BP & $\mathrm{AP}$ & $\mathrm{BP}$ & \\
\hline \multirow{6}{*}{ Mobile } & \multirow{3}{*}{5.67} & 20.684 & 20.093 & 22.725 & 19.155 & 100 \\
\hline & & 20.442 & 20.290 & 23.021 & 21.308 & 101 \\
\hline & & 20.971 & 20.790 & 22.967 & 20.380 & 102 \\
\hline & \multirow{3}{*}{3.4} & 19.529 & 18.683 & 22.374 & 18.321 & 100 \\
\hline & & 20.097 & 18.796 & 22.703 & 19.591 & 101 \\
\hline & & 20.812 & 18.797 & 22.485 & 18.891 & 102 \\
\hline \multirow{6}{*}{ Paris } & \multirow{3}{*}{5.67} & 27.455 & 23.945 & 27.725 & 22.429 & 100 \\
\hline & & 27.370 & 23.895 & 27.605 & 24.277 & 101 \\
\hline & & 27.403 & 22.668 & 27.224 & 23.896 & 102 \\
\hline & \multirow{3}{*}{3.4} & 26.626 & 22.198 & 27.169 & 21.300 & 100 \\
\hline & & 26.799 & 19.872 & 27.268 & 20.952 & 101 \\
\hline & & 27.333 & 22.213 & 26.983 & 21.794 & 102 \\
\hline
\end{tabular}

the condition of the channel. As the condition of the channel worsens (higher BER), more protection is required to protect the data.

\section{CONCLUSIONS}

In this paper, we have examined the transmission of MJPEG2000, encoded frames of video sequences. We presented a method for adaptively adding error protection by analyzing the condition of the channel and the frame and 


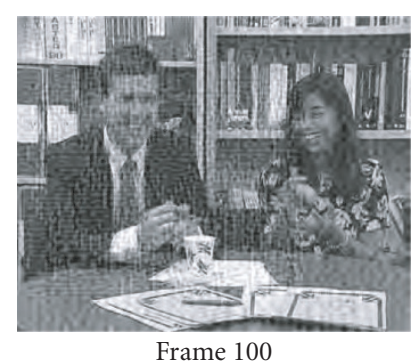

(a)

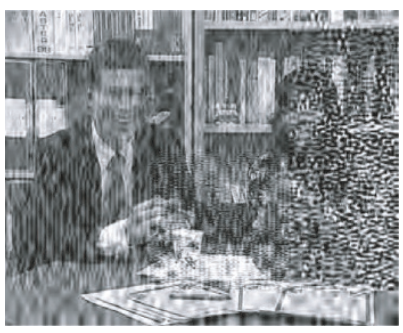

Frame 100

(d)

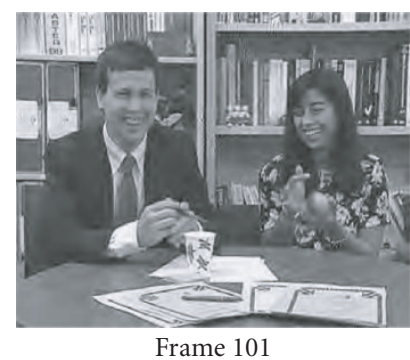

(b)

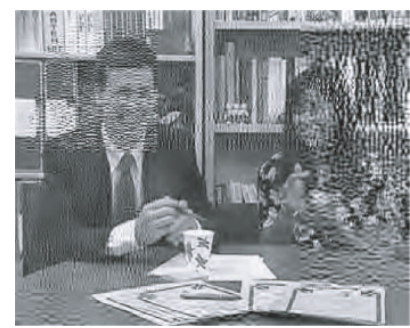

Frame 101

(e)

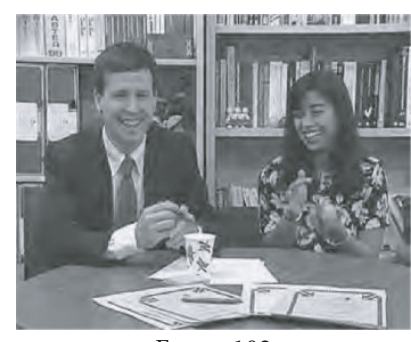

Frame 102

(c)

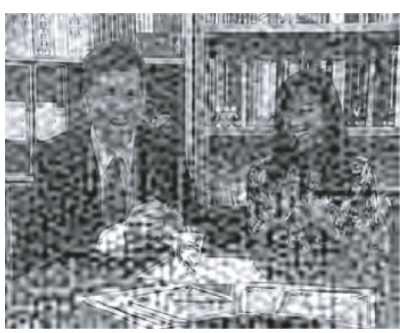

Frame 102

(f)

FIgUre 11: Decoded frames of Paris sequence using (a), (b), (c) adaptive protection and (d), (e), (f) basic protection, all transmitted over a single channel with a bandwidth of $45000 \mathrm{bps}(\tau \sim 5.67 \mathrm{~s})$.

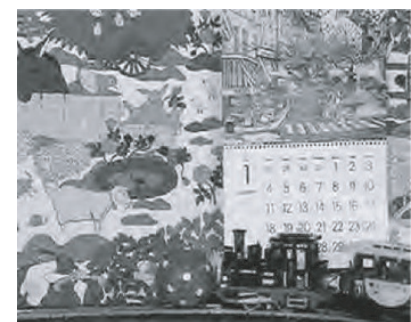

Frame 100

(a)

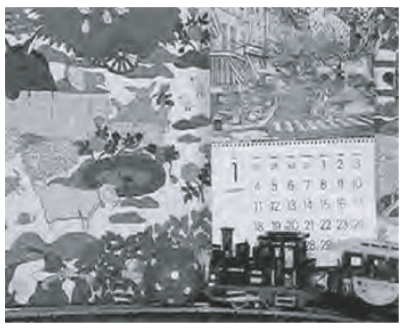

Frame 100

(d)

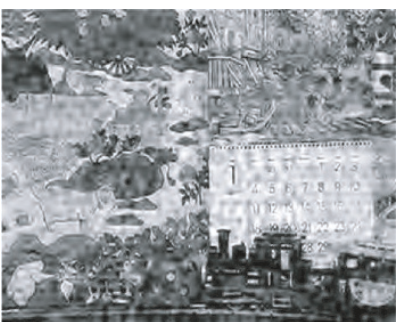

Frame 101

(b)

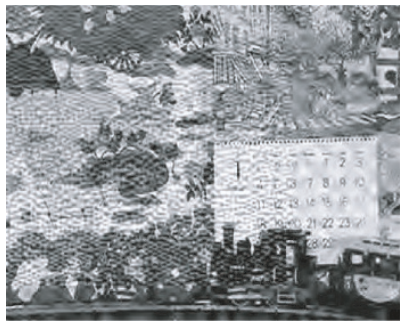

Frame 101

(e)

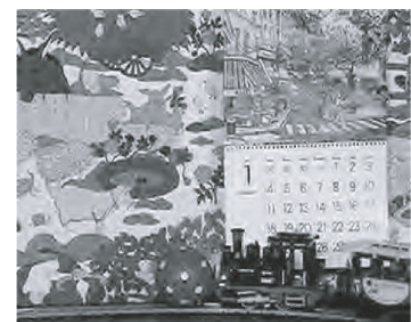

Frame 102

(c)

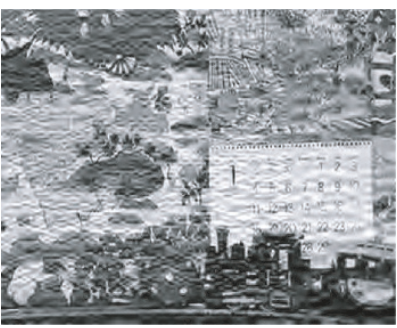

Frame 102

(f)

Figure 12: Decoded frames of Mobile sequence using (a), (b), (c) adaptive protection and (d), (e), (f) basic protection, all transmitted over a single channel with bandwidths of 42000 and 45000 bps $(\tau \sim 3.4$ s). 


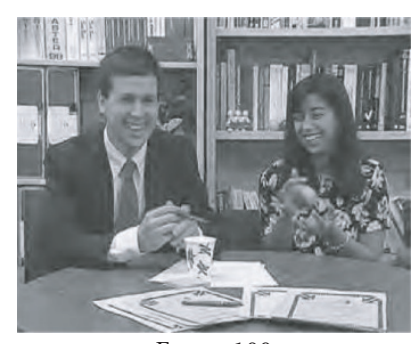

Frame 100

(a)

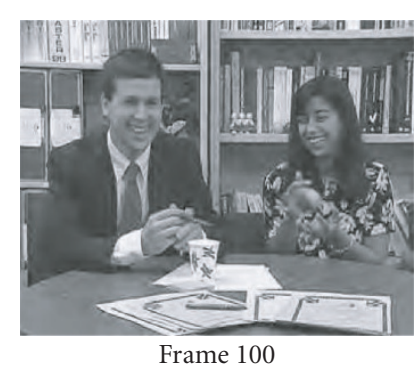

(d)

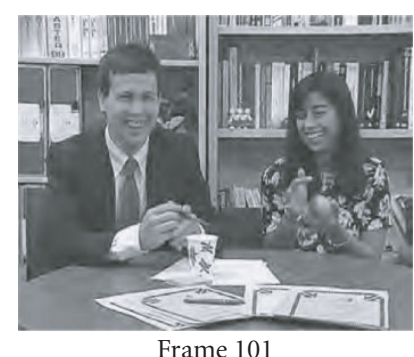

Frame 101

(b)

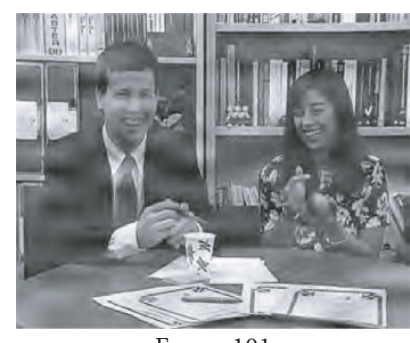

Frame 101

(e)

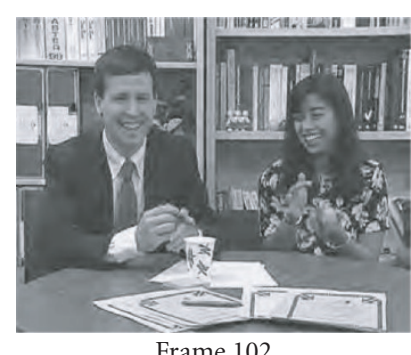

Frame 102

(c)

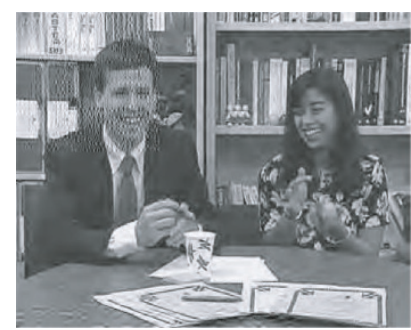

Frame 102

(f)

Figure 13: Decoded frames of Paris sequence using (a), (b), (c) adaptive protection and (d), (e), (f) basic protection, all transmitted over a single channel with bandwidths of 50000,40000 , and 35000 bps $(\tau \sim 5.67 \mathrm{~s})$.

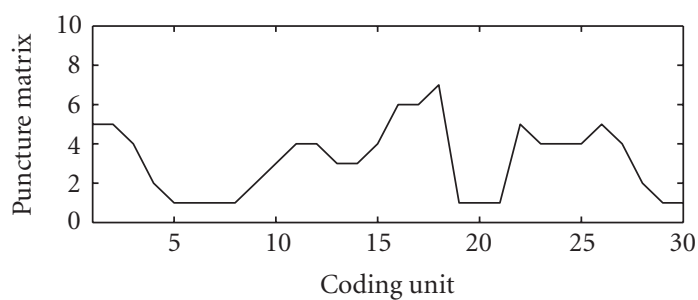

(a)

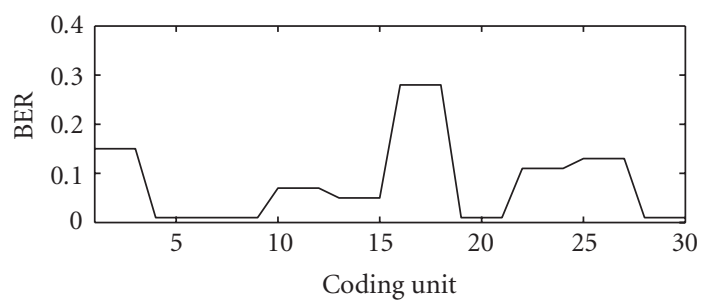

(b)

FIgURE 14: An example of time adaptivity of tiles over a single time-varying channel.

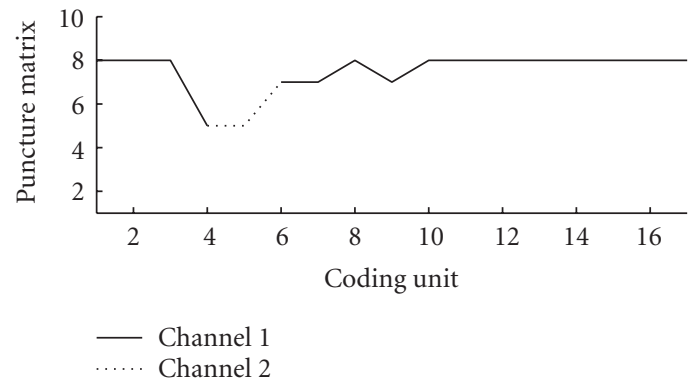

(a)

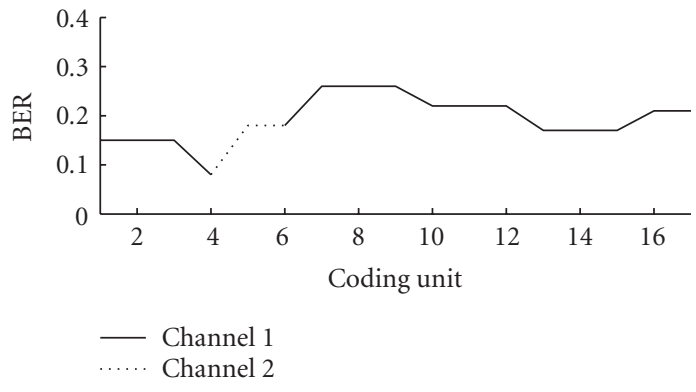

(b)

FIGURE 15: An example of time adaptivity of packets over two time-varying channels. 


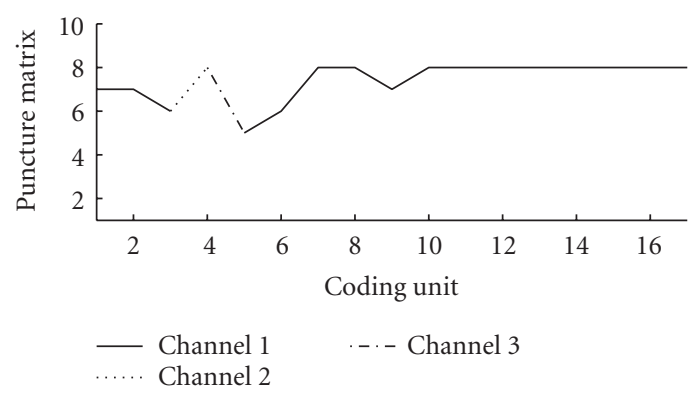

(a)

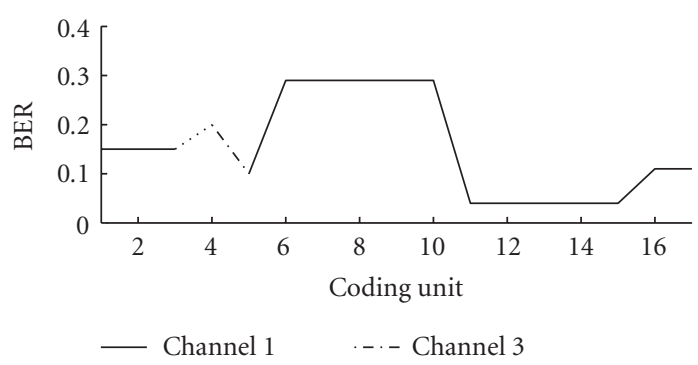

(b)

FIGURE 16: An example of time adaptivity of packets over three time-varying channels.

using a rate-flexible channel coder. In addition, we considered bandlimited channels, so there would be a tradeoff between the amount of reconstructed distortion and the allowable bit rate of the system. Frames from video sequences were encoded and transmission was simulated over errorprone channels. We showed that our method outperforms a method where the amount of protection available is constant regardless of the condition of the channel.

Of course, this method may not be advantageous in a real-time environment as each frame is encoded using MJPEG2000, which does not make use of efficient techniques of motion compensation. However, out method could be used effectively in a transmission situation which is not time-critical and where the end result is data storage (e.g.). In addition, the proposed method can be used in applications that require no error propagation when any frame is corrupted.

\section{REFERENCES}

[1] M. J. Ruf and J. W. Modestino, "Rate-distortion performance for joint source and channel coding of images," in Proceedings of IEEE International Conference on Image Processing (ICIP '95), vol. 2, pp. 77-80, Washington, DC, USA, October 1995.

[2] T. Chuu, Z. Liu, Z. Xiong, and X. Wu, "Joint UEP and layered source coding with application to transmission of JPEG-2000 coded images," in Proceedings of IEEE Global Telecommunications Conference (GLOBECOM '01), vol. 3, pp. 2036-2039, San Antonio, Tex, USA, November 2001.

[3] V. Chande and N. Farvardin, "Joint source-channel coding for progressive transmission of embedded source coders," in Proceedings of Data Compression Conference (DCC'99), pp. 52-61, Snowbird, Utah, USA, March 1999.

[4] P. G. Sherwood and K. Zeger, "Progressive image coding on noisy channels," in Proceedings of Data Compression Conference (DCC '97), pp. 72-81, Snowbird, Utah, USA, March 1997.

[5] B. A. Banister, B. Belzer, and T. R. Fischer, "Robust image transmission using JPEG2000 and turbo-codes," IEEE Signal Processing Letters, vol. 9, no. 4, pp. 117-119, 2002.

[6] S. Dumitrescu, X. Wu, and Z. Wang, "Globally optimal uneven error-protected packetization of scalable code streams," IEEE Transactions on Multimedia, vol. 6, no. 2, pp. 230-239, 2004.

[7] H. Li and C. W. Chen, "Robust image transmission with bidirectional synchronization and hierarchical error correction," IEEE Transactions on Circuits and Systems for Video Technology, vol. 11, no. 11, pp. 1183-1187, 2001.
[8] L. Cao and C. W. Chen, "Multiple hierarchical image transmission over wireless channels," in Proceedings of IEEE Wireless Communications and Networking Conference (WCNC'99), vol. 1, pp. 349-353, New Orleans, La, USA, September 1999.

[9] N. Chaddha and S. Diggavi, "A frame-work for joint sourcechannel coding of images over time-varying wireless channels," in Proceedings of IEEE International Conference on Image Processing (ICIP'96), vol. 2, pp. 89-92, Lausanne, Switzerland, September 1996.

[10] V. S. Sanchez and M. K. Mandal, "Robust transmission of JPEG2000 images over noisy channels," IEEE Transactions on Consumer Electronics, vol. 48, no. 3, pp. 451-456, 2002.

[11] J. Kim, R. M. Mersereau, and Y. Altunbasak, "Error-resilient image and video transmission over the Internet using unequal error protection," IEEE Transactions on Image Processing, vol. 12, no. 2, pp. 121-131, 2003.

[12] T. Fukuhara, K. Katoh, S. Kimura, K. Hosaka, and A. Leung, "Motion-JPEG2000 standardization and target market," in Proceedings of IEEE International Conference on Image Processing (ICIP '00), vol. 2, pp. 57-60, Vancouver, BC, Canada, September 2000.

[13] F. Dufaux and T. Ebrahimi, "Motion JPEG2000 for wireless applications," in Proceedings of 1st International JPEG2000 Workshop, pp. 2036-2039, Lugano, Switzerland, July 2003.

[14] S. Fossel, G. Fottinger, and J. Mohr, "Motion JPEG2000 for high quality video systems," IEEE Transactions on Consumer Electronics, vol. 49, no. 4, pp. 787-791, 2003.

[15] D. S. Taubman and M. W. Marcellin, JPEG2000: Image Compression Fundamentals, Standards and Practice, Kluwer Academic, Boston, Mass, USA, 2002.

[16] J. Hagenauer, "Rate-compatible punctured convolutional codes (RCPC codes) and their applications," IEEE Transactions on Communications, vol. 36, no. 4, pp. 389-400, 1988.

[17] S. Coleri, M. Ergen, A. Puri, and A. Bahai, "Channel estimation techniques based on pilot arrangement in OFDM systems," IEEE Transactions on Broadcasting, vol. 48, no. 3, pp. 223-229, 2002.

[18] T. P.-C. Chen and T. Chen, "Adaptive joint source-channel coding using rate shaping," in Proceedings of IEEE International Conference on Acoustics, Speech, and Signal Processing (ICASSP '02), vol. 2, pp. 1985-1988, Orlando, Fla, USA, May 2002.

[19] L. P. Kondi, F. Ishtiaq, and A. K. Katsaggelos, "Joint sourcechannel coding for motion-compensated DCT-based SNR scalable video," IEEE Transactions on Image Processing, vol. 11, no. 9, pp. 1043-1052, 2002. 
[20] A. Ortega and K. Ramchandran, "Rate-distortion methods for image and video compression," IEEE Signal Processing Magazine, vol. 15, no. 6, pp. 23-50, 1998.

[21] M. Bystrom and J. W. Modestino, "Combined source-channel coding schemes for video transmission over an additive white Gaussian noise channel," IEEE Journal on Selected Areas in Communications, vol. 18, no. 6, pp. 880-890, 2000.

Scott Bezan received his Bachelor of Engineering degree in electrical engineering from McMaster University in Hamilton, Ontario, Canada, in 2002, and his Master of Applied Science degree in electrical engineering also from McMaster in 2004. During his graduate education, Scott's research focused on image and video processing and error correction coding involved in robust multimedia transmission.

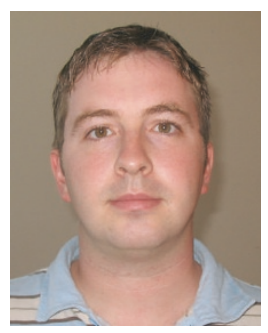

Shahram Shirani received his Bachelor of Engineering degree in electrical engineering from Isfahan University of Technology, Iran, and his Master of Science (with honor) degree in biomedical engineering from Amirkabir University of Technology, Iran, and his Ph.D. degree in electrical engineering from University of British Columbia, Canada, in 1989, 1994, and 2000, respectively. Since July 2000, he has been

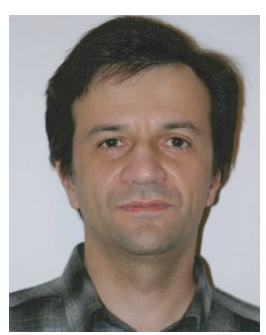
with the Department of Electrical and Computer Engineering, McMaster University, as an Assistant Professor. His research interests are mainly focused on image and video processing, multimedia compression and communications, medical image processing and hardware architectures for image and video processing. He has published more than 60 journal and conference papers. He is a Senior Member of IEEE, a Member of Technical Program Committee of ICIP, a Member of Technical Program Committee of ICASSP, and a Professional Engineer. 